\title{
Exceso de peso corporal en estudiantes universitarios según variables sociodemográficas y estilos de vida
}

\section{Excess weight body in university students by sociodemographic and lifestyle variables}

\begin{abstract}
Introduction: University students are particularly vulnerable to inadequate food intake, which has consequences for their nutritional and health status. Objective: To study the prevalence of overweight (OW) and its associated factors among a group of students at University of Antioquia, Colombia. Materials and Methods: Cross-sectional study involving 424 male and female students $21.4 \pm 4.0$ years of age. OW was diagnosed by body mass index $(B M I) \geq 25$ $\mathrm{kg} / \mathrm{m} 2$; risk of central adiposity was measured by waist circumference $(W C) \geq 90 \mathrm{~cm}$ and $\geq 80 \mathrm{~cm}$ in men and women, respectively. Socio-demographic and lifestyle variables were measured. Chi2 test $(\alpha=0.05)$ was used as a descriptive statistic. Results: $17.1 \%$ of students had OW, which was higher in men (men: $22.6 \%$ vs women $14.0 \%$, p<0.05); $8.1 \%$ had risk of central adiposity higher, which was higher in women (men: $6.5 \%$ vs women: $9.1 \%, p>$ $0.05)$. BMI was associated with sex $(p=0.001)$. Risk of central adiposity was not related with socio-demographic or lifestyle characteristics. Conclusion: A considerable percentage of university students were overweight based on BMI, which related to sex of the student. However, it is important to promote university spaces that are conducive to healthy lifestyles.
\end{abstract}

Key words: Obesity, overweight, waist circumference, body mass index, university students.

\section{INTRODUCCIÓN}

En las últimas décadas, las prevalencias de sobrepeso y obesidad muestran una tendencia ascendente a nivel mundial, cuyo impacto económico y social supone un creciente problema de salud pública'. Según Popkin y cols², para el año 2030, aproximadamente 2,16 billones de adultos de todo el mundo tendrán sobrepeso y 1,12 billones serán obesos debido a los dramáticos cambios en la alimentación. Esta situación constituye un factor de riesgo de padecer diversos

\author{
Laura González-Zapata ${ }^{1,2,3}$, Cristina \\ Carreño-Aguirre ${ }^{1,2}$, Alejandro Estrada ${ }^{1,2,4}$, \\ Julia Monsalve-Alvarez ${ }^{1,2}$, \\ Luz Stella Alvarez ${ }^{1,2,3}$
}

1. Universidad de Antioquia. 2. Escuela de Nutrición y Dietética. 3. Grupo de Investigación en Determinantes Sociales y Económicos de la situación de salud y nutrición. 4. Grupo de investigación en demografía y salud

Dirigir correspondencia a: Cristina Carreño Aguirre, Nutricionista Dietista. Calle 49 D sur \# 40 A 78. Medellín, Colombia Teléfono: 3003403390/3328403.

Email: cristina.carreno@udea.edu.co

Este trabajo fue recibido el 26 de enero de 2017. Aceptado con modificaciones: 26 de abril de 2017. Aceptado para ser publicado: 28 de junio de 2017.

problemas de salud como enfermedades cardiovasculares, desórdenes metabólicos, diabetes mellitus, complicaciones ortopédicas y algunos tipos de cáncer ${ }^{1-4}$.

En los países de ingresos altos, la obesidad se presenta en mayor medida en el nivel socioeconómico bajo y es considerada una enfermedad de alto costo sanitario ${ }^{5-7}$. En los de ingresos bajos y medianos, junto con una mayor velocidad en el incremento de las tasas, también se ha observado diferencias según el nivel socioeconómico ${ }^{2,6}$. 
En Colombia, el 44,2\% de la población entre 18 y 42 años de edad presentó algún grado de exceso de peso (EP), según la Encuesta Nacional de la Situación Nutricional 2010 $(\text { ENSIN })^{8}$, mientras que en Medellín fue del $42,6 \%$ en un grupo de edad similar (18 a 44), según los datos del Perfil Alimentario y Nutricional de los hogares de Medellín y sus corregimientos 2010 (PAYNM) ${ }^{9}$.

Diversos autores han destacado que la población universitaria es un grupo especialmente vulnerable desde el punto de vista nutricional, y las alteraciones en su peso corporal se han relacionado fuertemente con su estilo de vida $^{10-14}$. Los comportamientos poco saludables son las principales causas de la morbilidad y mortalidad prematura en este grupo: la inactividad física, el tabaquismo, el consumo de alcohol y la mala calidad de la alimentación, son los cuatro principales contribuyentes al desarrollo de obesidad, enfermedades cardiovasculares, cáncer y diabetes ${ }^{12-17}$.

Investigaciones sobre estilos de vida reportan importantes prevalencias de consumo de tabaco y alcohol en los universitarios. El Reporte de uso de Drogas en Colombia del 2015 encontró que, en este grupo, el consumo de alcohol aumentó desde el 2008 casi en cuatro puntos porcentuales y alcanzó un $84,76 \%$ en el 2013, en el mismo año el consumo de tabaco fue de $29,37 \%{ }^{18}$. De otro lado, la mayor tasa de uso de alcohol se presentó entre jóvenes de 18 a 24 años, seguidos del grupo de 25 a 34 años $^{19}$. En cuanto al tabaco/ cigarrillo, el consumo crece a partir de los 18 años, con una prevalencia cercana al $16 \%$, entre los 18 y 34 años de edad ${ }^{19}$. Por su parte en Medellín, las prevalencias de último mes o consumo actual de cigarrillo de este mismo grupo de edad fue del $39,4 \%$ y el uso de alcohol fue del $46,7 \%{ }^{19}$, por tanto, Medellín y su Área metropolitana es la segunda ciudad de todo el país con el mayor uso de alcohol y cigarrillo en este grupo de $\operatorname{edad}^{20}$ y fue la cuarta ciudad con mayor consumo en universitarios ${ }^{21}$. Otros estudios realizados en Medellín y Bogotá, no hallaron diferencias por sexo en el uso de tabaco y alcohol en dicho grupo ${ }^{22,23}$.

En cuanto al hábito sobre práctica de actividad física, los estudios revelan bajos índices de práctica en población universitaria, en mayor proporción en mujeres ${ }^{10,24,25}$. En Medellín, tan solo el $16,5 \%$ de la población de 18 a 64 años cumple con las recomendaciones de actividad física (> 150 minutos acumulados por semana $)^{8}$.

A pesar del aumento progresivo en el mundo de las prevalencias de exceso de peso y la amplia documentación existente sobre las consecuencias asociadas a hábitos poco saludables, existe evidencia que demuestra que hay una alta proporción de adultos jóvenes, en especial estudiantes universitarios, que asumen este tipo de comportamientos nocivos para su salud ${ }^{10,14}$. Lo anterior, da cuenta de la necesidad de estudiar qué tipo de factores sociales y económicos, así como que comportamientos relacionados con estilos de vida se ven más asociados al exceso de peso en esta población.

El objetivo de este estudio es investigar la frecuencia de exceso de peso según índice de masa corporal (IMC), riesgo cardiovascular por perímetro de cintura $(\mathrm{PC})$ y riesgo de adiposidad en análisis conjunto de IMC y PC, en estudiantes de la Universidad de Antioquia de MedellínColombia y su relación con factores sociodemográficos y variables propias de estilos de vida.

\section{MATERIALES Y MÉTODOS}

Estudio descriptivo transversal, que tuvo como población de referencia los 36.500 estudiantes que en promedio, aparecían matriculados para el período de estudio en la Universidad de Antioquia (UdeA). Como mecanismo de reclutamiento, se invitó a participar a todos los estudiantes que asistieron a las jornadas de salud lideradas por Bienestar Universitario en todas las dependencias que componen la comunidad académica de la UdeA (ciencias sociales y humanas, ciencias exactas y naturales, y ciencias de la salud). Un total de 424 estudiantes que asistieron a las jornadas, aceptaron participar de manera voluntaria en el estudio.

Los criterios de inclusión consideraron estudiantes de ambos sexos, con matrícula vigente en la UdeA, que no presentaran impedimentos para la toma de medidas antropométricas y que aceptaran participar en la investigación mediante la firma del consentimiento informado. En el caso de las mujeres se tuvo como criterios de exclusión estar embarazadas o en periodo de lactancia materna. Los datos se recolectaron entre marzo de 2009 y diciembre de 2010.

Las mediciones antropométricas tomadas fueron el peso en $\mathrm{kg}$, la estatura y el PC en cm. Para la medición del peso corporal se utilizó una báscula digital marca Tanita HD314 con una capacidad de $150 \mathrm{~kg}$ y $0,1 \mathrm{~kg}$ de sensibilidad; la técnica de medición contempló que la balanza estuviera sobre una superficie firme y equilibrada en cero, posteriormente se pidió a los participantes que se situaran directamente en la mitad de la superficie de la balanza con ropa liviana y la mínima cantidad de accesorios, luego se esperó a que se estabilizara la balanza en el valor de la medida. La estatura se midió con un metro portátil marca SECA con cinta métrica metálica, cuerpo en pasta, pieza fija y escuadra móvil, con una longitud de $220 \mathrm{~cm}$ y una sensibilidad de $0,1 \mathrm{~cm}$; las personas se midieron sin zapatos y sin accesorios en la cabeza situándose con los pies sobre las plantillas en posición anatómica debajo del metro portátil y con la espalda tocando la pared. Así mismo, para producir una medida más consistente, la cabeza del sujeto estuvo colocada en el plano de Frankfort y se instruyó al mismo que en el momento de la medida tomara una inspiración profunda para que la columna permaneciera lo más erguida posible.

El PC se midió con una cinta métrica (MABIS) no extensible, con una longitud de $150 \mathrm{~cm}$ y una sensibilidad de $0,1 \mathrm{~cm}$, en el proceso se situó al sujeto en posición anatómica con el abdomen relajado y los brazos cruzados sobre el pecho, seguidamente la medida se tomó palpando y marcando la última costilla y el borde superior de la cresta ilíaca de ambos costados, y en el punto medio se ubicó la cinta métrica sin presionar el tejido de la piel; la lectura se realizó al final de una espiración normal. Para la validación cada una de las 
medidas anteriormente descritas se tomó dos veces, cuando la diferencia en peso superó los $100 \mathrm{~g}$ se procedió a una tercera medición; en la estatura y el PC diferencias mayores a 5 milímetros requirieron una tercera toma de la medida.

La recolección de los datos estuvo a cargo de estudiantes de Nutrición y Dietética, previamente capacitados y estandarizados en el proceso de investigación y en la toma de medidas antropométricas. En la valoración de los datos antropométricos, se descartó en IMC a cuatro participantes (mujeres) de las que no fue posible obtener el peso. En el análisis por riesgo cardiovascular se excluyó a cinco mujeres y un hombre por no tener el dato de PC.

A partir de los datos de peso y talla se calculó el IMC como el cociente entre peso en $\mathrm{kg} / \mathrm{talla}$ en $\mathrm{m}^{2}$, y se interpretó en adultos según los criterios de la Organización Mundial de la Salud: bajo peso $\left(<18,5 \mathrm{~kg} / \mathrm{m}^{2}\right)$, normalidad $(18,5$ a 24,9 $\left.\mathrm{kg} / \mathrm{m}^{2}\right)$, sobrepeso $\left(25,0\right.$ a $\left.29,9 \mathrm{~kg} / \mathrm{m}^{2}\right)$ y obesidad $(\geq 30 \mathrm{~kg} /$ $\mathrm{m}^{2}$ ). En el caso de los menores de 18 años, la interpretación se realizó según lo establecido en la Resolución Colombiana 2121 de $2010^{26}$. Para efectos de este estudio, se empleó la categoría de exceso de peso para los valores de IMC $\geq 25^{27}$.

Los datos del PC se clasificaron como riesgo de enfermedad cardiovascular, si los valores eran $\geq 80 \mathrm{~cm}$ para mujeres y $\geq 90 \mathrm{~cm}$ para hombres ${ }^{28}$. Como medida complementaria, se retomó la experiencia canadiense, que empleó el índice de adiposidad, basado en un análisis conjunto a partir del IMC y el PC ${ }^{12}$ (Tabla 1).

\section{Variables explicativas}

Los estudiantes participantes dieron respuesta a un cuestionario estructurado con preguntas cerradas y dirigido por un encuestador, que incluyó variables sociodemográficas y relacionadas con estilos de vida, a saber:

1) Variables sociodemográficas: edad en años cumplidos; sexo; la condición de estudiante trabajador al momento de la realización de la encuesta (Si/No); área de formación profesional (ciencias de la salud, ciencias sociales y humanas, y ciencias exactas y naturales); estrato socioeconómico de la vivienda (en Colombia el DANE clasifica la población en seis grupos, de acuerdo a las características de las viviendas y el entorno social, donde uno es el estrato socioeconómico más bajo y seis el más alto), para efectos de este estudio, se agrupó la variable en tres sub-categorías: estrato bajo 1 y 2 , estrato medio 3 y 4 , estrato alto 5 , ya que no hubo participantes de estrato 6 .

2) Variables relacionadas con estilos de vida: (a) Hábito de fumar: si, nunca y ex fumador (que no fumó en los últimos 6 meses). Si la respuesta fue afirmativa se indagó por la edad de inicio y la cantidad consumida a diario (si era el caso); (b) Consumo de bebidas alcohólicas alguna vez en la vida: si, no, y ex consumidor. Si la respuesta fue afirmativa se indagó por la frecuencia de consumo en los últimos 12 meses, así como la cantidad de copas o unidades de alcohol; (c) Actividad principal de la vida diaria: i) sentado/a la mayor parte de la jornada, ii) caminando, caminando llevando algún peso, efectuando desplazamientos frecuentes, iii) de pie la mayor parte de la jornada sin efectuar grandes desplazamientos o esfuerzos, y iv) realizando tareas que requieran gran esfuerzo físico y (d) Horas dedicadas a ver televisión al día.

Los datos se procesaron en el software SPSS versión 18 para Windows (Chicago: SPSS Inc.; 2010). Se obtuvo el cálculo de medidas descriptivas: promedios, desviaciones, percentiles y porcentajes y se utilizó la prueba de $\mathrm{Chi}^{2} \mathrm{o}$ la prueba exacta de Fisher, para comprobar asociación entre las variables de IMC, PC y adiposidad con las demás variables sociodemográficas y de estilos de vida. El nivel de significancia estadística fue de 0,05.

\section{Consideraciones éticas}

Este proyecto fue avalado el 5 febrero de 2009 según consta en acta 09-043-208, por el Comité de Bioética de la Sede de Investigación Universitaria (SIU) de la UdeA, como "investigación con riesgos menores al mínimo".

\begin{tabular}{|c|c|c|c|}
\hline \multicolumn{4}{|c|}{$\begin{array}{c}\text { Tabla } 1 \\
\text { Clasificación del riesgo de adiposidad de acuerdo al índice de masa corporal y perímetro de cintura }\end{array}$} \\
\hline Perímetro de cintura & $\begin{array}{c}\text { Normal } \\
(\text { IMC 18,5 - 24,9) }\end{array}$ & $\begin{array}{c}\text { Sobrepeso } \\
(\text { IMC 25,0 - 29,9) }\end{array}$ & $\begin{array}{l}\text { Obesidad } \\
(\mathrm{IMC} \geq 30)\end{array}$ \\
\hline $\begin{array}{l}<90 \mathrm{~cm} \text { (hombres) } \\
<80 \mathrm{~cm} \text { (mujeres) }\end{array}$ & Sin riesgo & Riesgo incrementado & Alto riesgo \\
\hline $\begin{array}{l}\geq 90 \mathrm{~cm} \text { (hombres) } \\
\geq 80 \mathrm{~cm} \text { (mujeres) }\end{array}$ & Riesgo incrementado & Alto riesgo & Alto riesgo \\
\hline
\end{tabular}




\section{RESULTADOS}

Se evaluaron 424 estudiantes con edades entre 17 y 42 años $(21,4 \pm 4,1)$. El promedio de edad en años para las mujeres fue $21,0 \pm 3,0$, y el de los hombres $22,0 \pm 5,0$. El $63,4 \%$ de los participantes fueron del sexo femenino $(n=269)$. El $60,6 \%$ de los estudiantes manifestó que sus viviendas estaban ubicadas en los estratos socioeconómicos 3 y 4. Respecto al área de formación, la mayor parte $48,8 \%$ fue del área de las ciencias de la salud (Tabla 2).

En las variables relacionadas con el estilo de vida de los universitarios, un 9,9\%, manifestaron fumar y de estos, un 40,5\% lo realizó todos los días. En cuanto a la ingesta de alcohol, el 74,5\% reportó su consumo en los últimos 12 meses y de estos, un $18,7 \%$ manifestó un consumo semanal con ingestas hasta de 7 o más copas o unidades de alcohol en un $33,8 \%$ de los casos (Tabla 2). Entre quienes consumen alcohol, las mujeres presentaron el mayor consumo $(61,4 \%)$.

En cuanto a los estilos de vida se encontró un predominio de conductas sedentarias: el $58,7 \%$ de los estudiantes no realizó ninguna actividad física o fueron de intensidad leve y el $46,2 \%$ reportó pasar sentado la mayor parte de la jornada. Con respecto al hábito de ver televisión, un 23,3\% reportó verla a diario (Tabla 2), con una duración hasta de más de dos horas en el 38,4\% de los casos (datos no mostrados).

El promedio de IMC para las mujeres fue 21,8 $8 \pm 3,4$ y el de los hombres 22,6 $6 \pm 3,1$; el porcentaje de exceso (sobrepeso más obesidad) por IMC fue del 17,1\% (IC95\%: $13,6 \% ; 20,8 \%$ ), siendo mayor en los hombres que en las mujeres ( $22,6 \%$ vs $14,0 \%)$. El déficit de peso se encontró en un $8,1 \%$ (IC95\%: 5,4\%; 10,6\%) siendo mayor en las mujeres $(11,3 \%$ vs $2,6 \%$ ). Por su parte, el PC promedio fue $69,9 \pm 8,0$ en las mujeres y de $76,7 \pm 8,0$ en los hombres; el porcentaje de estudiantes con riesgo de enfermedad cardiovascular por este indicador fue de 8,1\% (IC95\%: 5,4\%; 10,6\%), más alta en las mujeres $(9,1 \%$ vs $6,5 \%)$ (Tablas 2 y 3 ). Cabe destacar que, del total de estudiantes con riesgo por PC, el 87,9\% presentó exceso de peso por IMC. Así mismo, de aquellos universitarios con sobrepeso y obesidad, el $32,2 \%$ y el $100 \%$ respectivamente, coincidió en tener riesgo de enfermedad cardiovascular por PC.

El porcentaje de estudiantes con riesgo global de adiposidad fue de $17,3 \%$ (IC95\%: $13,6 \% ; 20,8 \%$ ), sin diferencias significativas según las variables socio demográficas y relacionadas con estilos de vida ( $p>0,05)$, con excepción de la distribución por sexo, donde se encontró mayor riesgo de adiposidad entre los hombres $(22,7 \% ; p=0,025)$ (Tabla 3$)$.

En relación al IMC solo se encontró asociación con el sexo $(p=0,001)$ (Tabla 4). El riesgo de enfermedad cardiovascular fue del 8,0\% y no mostró asociación con las características sociodemográficas y de estilo de vida analizadas ( $p>0,05)$ (Tabla 5).

\section{DISCUSIÓN}

Son escasos los estudios publicados sobre el exceso de peso de los estudiantes universitarios y su relación con variables sociodemográficas y de estilos de vida, especialmente en la ciudad de Medellín. El presente estudio muestra que casi una quinta parte de la población universitaria de la Universidad de Antioquia presentó exceso de peso según IMC, con diferencias estadísticamente significativas según sexo.

Aspectos relacionados con estilos de vida

A pesar de que en nuestros resultados se encontró un bajo porcentaje de consumo de tabaco comparado con otros estudios nacionales ${ }^{18,23}$ y regionales ${ }^{20,21,29}$, de aquellos estudiantes que manifestaron fumar (9,9\%), un 40,5\% fumó todos los días. Estos hallazgos requieren de especial atención pues, se ha definido que el consumo de tabaco es la causa principal de muerte evitable en el mundo ${ }^{30}$. Además, los estilos de vida poco saludables como el consumo de alcohol y tabaco son factores de riesgo de alteraciones en el estado de salud y nutrición. Lo anterior, sumado a la disminución de la actividad física, cambios en los hábitos de vida y de trabajo, puede impactar negativamente el estado nutricional y el peso corporal de los estudiantes ${ }^{10,11,17}$.

Por su parte, en los universitarios encuestados, la proporción de consumo de bebidas alcohólicas durante los últimos 12 meses es alta (74,5\%). Este resultado se asemeja a la tendencia de consumo observada por estudios de nivel nacional. En el informe PRADICAN y el Reporte de Drogas se encontró que el $84,8 \%$ de los universitarios consumieron alcohol durante el último año ${ }^{18,23}$. Así mismo, Ilama la atención el hecho de que, en términos de la edad, los más jóvenes presenten la mayor tasa de uso actual de alcohol con respecto a los demás grupos de población ${ }^{19}$. Esta diferencia es más notoria en el departamento de Antioquia alcanzando un 52,0\% para jóvenes de 18 a 24 años $^{20}$. En este sentido, en la UdeA aquellos más jóvenes pueden ser más vulnerables a un mayor consumo; por lo tanto, es importante evaluar si ese consumo puede ser perjudicial para la salud de los estudiantes.

Estudios realizados en ciudades principales de Colombia, determinaron mayor consumo de alcohol en los hombres ${ }^{21,31}$, siendo contrario a nuestros resultados (mujeres 61,4\%; hombres 38,6\%). Esto podría ser explicado a la luz de algunos trabajos que sugieren que el incremento del consumo de alcohol por parte del sexo femenino se puede relacionar con características socioculturales como la mayor aceptación hacia la participación de su género en los espacios sociales y recreativos ${ }^{22}$ en donde las más propensas al consumo son las jóvenes de veinte años y ya cerca de los treinta ${ }^{32}$, que constituyen el grupo de edad de mayor peso en este estudio.

Según los hallazgos mencionados, es importante prestar atención al consumo del alcohol de los universitarios, sobre todo si se tiene en cuenta que en los últimos años se ha evidenciado que el consumo de alcohol probablemente ha contribuido al exceso en la ingesta de energía que se asocia con el aumento de peso ${ }^{33}$.

En cuanto a la práctica de actividad física, más del $50 \%$ de los estudiantes de la UdeA no realizaron ninguna actividad o desarrollaron actividades con intensidad leve. Este 
Tabla 2

Caracterización de la población de estudio según variables analizadas

\begin{tabular}{|c|c|c|c|}
\hline \multicolumn{2}{|l|}{ Características } & \multirow{2}{*}{$\begin{array}{c}\mathbf{n} \\
269\end{array}$} & \multirow{2}{*}{$\begin{array}{c}\% \\
63,4\end{array}$} \\
\hline Sexo & Mujer & & \\
\hline & Hombre & 155 & 36,6 \\
\hline \multirow[t]{4}{*}{ Edad } & $<18$ & 36 & 8,5 \\
\hline & $18-22$ & 279 & 65,8 \\
\hline & $23-27$ & 76 & 17,9 \\
\hline & $>27$ & 33 & 7,8 \\
\hline \multirow[t]{3}{*}{ Estrato socioeconómico } & $1-2$ & 151 & 35,6 \\
\hline & $3-4$ & 257 & 60,6 \\
\hline & 5 & 16 & 3,8 \\
\hline \multirow[t]{3}{*}{ Área de formación } & Ciencias de la Salud & 207 & 48,8 \\
\hline & Ciencias Sociales y Humanas & 157 & 37,0 \\
\hline & Ciencias Exactas y Naturales & 60 & 14,2 \\
\hline \multirow[t]{3}{*}{ Hábito de fumar } & $\mathrm{Si}$ & 42 & 9,9 \\
\hline & Nunca & 345 & 81,4 \\
\hline & Ex fumador & 37 & 8,7 \\
\hline \multirow[t]{3}{*}{ Consumo de alcohol alguna vez en la vida } & Si y aun consume & 316 & 74,5 \\
\hline & No & 98 & 23,1 \\
\hline & Ex consumidor & 10 & 2,4 \\
\hline \multirow[t]{4}{*}{ Usualmente en la semana, su actividad física es } & Ligera o leve & 137 & 32,3 \\
\hline & Moderada & 128 & 30,2 \\
\hline & Intensa & 47 & 11,1 \\
\hline & Ninguno & 112 & 26,4 \\
\hline \multirow[t]{4}{*}{ Actividad principal de su vida diaria* } & 1 & 196 & 46,2 \\
\hline & 2 & 166 & 39,2 \\
\hline & 3 & 50 & 11,8 \\
\hline & 4 & 12 & 2,8 \\
\hline \multirow[t]{2}{*}{ Ver Televisión a diario } & Si & 99 & 23,3 \\
\hline & No & 325 & 76,7 \\
\hline \multirow[t]{2}{*}{ Trabaja en la actualidad } & $\mathrm{Si}$ & 115 & 27,1 \\
\hline & No & 309 & 72,9 \\
\hline \multirow[t]{3}{*}{ Índice de masa corporal } & Bajo & 34 & 8,1 \\
\hline & Normal & 314 & 74,8 \\
\hline & Exceso & 72 & 17,1 \\
\hline \multirow[t]{2}{*}{ Perímetro de Cintura } & Riesgo Enfermedad Cardiovascular & 34 & 8,1 \\
\hline & Normal & 384 & 91,9 \\
\hline \multicolumn{4}{|c|}{ *1. Sentado/a la mayor parte de la jornada } \\
\hline \multirow{2}{*}{\multicolumn{4}{|c|}{$\begin{array}{l}\text { 2. Caminando, caminando llevando algún peso, efectuando desplazamientos frecuentes } \\
\text { 3. De pie la mayor parte de la jornada sin efectuar grandes desplazamientos o esfuerzos }\end{array}$}} \\
\hline & & & \\
\hline \multicolumn{4}{|c|}{ 4. Realizando tareas que requieran gran esfuerzo físico } \\
\hline
\end{tabular}


Tabla 3

Riesgo de adiposidad de los estudiantes según variables de interés.

Riesgo de Adiposidad

\section{Características}

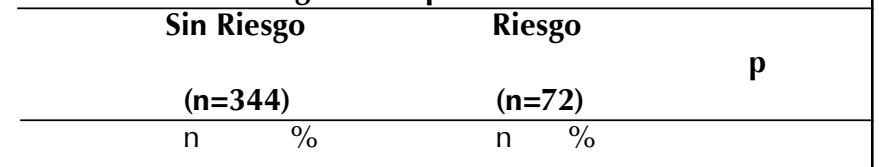

Edad

$$
<18
$$

$18-22$

$23-27$

$$
>27
$$

\section{Estrato}

socioeconómico

$$
\begin{aligned}
& 1-2 \\
& 3-4 \\
& 5
\end{aligned}
$$

Sexo

Masculino

Femenino

28

228

60

28

80,0

82,9

82,2

84,8

126

203

84,0

81,2

15

93,8

Ciencias de la Salud

Ciencias Sociales y Humanas

Ciencias Exactas y Naturales

$$
\text { Si }
$$

No

Si

Nunca

Ex fumador

Si y aun consume

No

Ex consumidor

alguna vez en la vida

\section{Ex consumidor}

Actividad principal de 1

s u vida diaria* 2

$$
3
$$

Ver Televisión a diario Si

$$
\begin{aligned}
& \text { Si } \\
& \text { No }
\end{aligned}
$$

225

77,3

85,9

$167 \quad 81,9$

12581,7

5288,1

94

250

85,5

81,7

$$
31
$$

283

77,5

83,5

$30 \quad 81,1$

251

86

80,7

89,6

77,8

158

82,7

138

84,7

78,0

975,0

$\begin{array}{rr}80 & 82,5 \\ 264 & 82,8\end{array}$

264
82,8

$\begin{array}{ll}7 & 20,0\end{array}$

$47 \quad 17,1$

$13 \quad 17,8$

$5 \quad 15,2$

0,959*

$24 \quad 16,0$

$47 \quad 18,8$

16,3

$0,429^{* *}$

$\begin{array}{ll}35 & 22,7\end{array}$

$37 \quad 14,1$

$37 \quad 18,1$

$28 \quad 18,3$

$\begin{array}{ll}7 & 11,9\end{array}$

0,490 *

$16 \quad 14,5$

$56 \quad 18,3$

$0,372 *$

922,5

$56 \quad 16,5$

$7 \quad 18,9$

$0,616^{*}$

$0,025^{*}$

$60 \quad 19,3$

$10 \quad 10,4$

222,2

$0,089 * *$

$33 \quad 17,3$

$25 \quad 15,3$

1122,0

325,0

$0,561^{* *}$

$17 \quad 17,5$

$55 \quad 17,2$

* 1 Sentado/a la mayor parte de la jornada

2 Caminando, caminando llevando algún peso, efectuando desplazamientos frecuentes

3 De pie la mayor parte de la jornada sin efectuar grandes desplazamientos o esfuerzos

4 Realizando tareas que requieran gran esfuerzo físico.

* Prueba Chi cuadrado; ** Prueba exacta de Fisher 
Tabla 5

Clasificación del perímetro de cintura según variables de interés

Perímetro de Cintura

Características

\begin{tabular}{ccc|}
\hline $\begin{array}{c}\text { Riesgo Enfermedad } \\
\text { Cardiovascular } \\
(n=34)\end{array}$ & Normal & $p$ \\
\hline $\mathbf{n} \%$ & $\mathbf{n} \quad \%$ & \\
\hline
\end{tabular}

Edad

$<18$
$18-22$
$23-27$
$>27$

4

11,1

32

88,9

$\begin{array}{rr}18 & 6,5 \\ 8 & 11,0\end{array}$

258

93,5

$0,317^{* *}$

65

89,0

$4 \quad 12,1$

29

87,9

Estrato socioeconómico

$$
\begin{aligned}
& 1-2 \\
& 3-4 \\
& 5
\end{aligned}
$$

$\begin{array}{rr}7 & 4,7 \\ 26 & 10, \\ 1 & 6,3\end{array}$

143

226

95,3

0,3

15

89,7

$0,116^{* *}$

Sexo

Masculino

$10 \quad 6,5$

144

93,8

Femenino

24

240

93,5

$0,349^{*}$

Área de formación

Ciencias de la Salud

20

9,1

90,9

Ciencias Sociales y Humanas

9,8

185

90,2

Ciencias Exactas y Naturales

8,5

140

91,5

5998,3

Trabaja en la actualidad $\quad \mathrm{Si}$

$$
\begin{aligned}
& \text { Si } \\
& \text { No }
\end{aligned}
$$

$\begin{array}{rr}9 & 8,1 \\ 25 & 8,1\end{array}$

102

91,9

$282 \quad 91,9$

Hábito de fumar

Si
Nunca
Ex fumador

$5 \quad 12,2$

$27 \quad 7,9$

$2 \quad 5,4$

36

313

87,8

92,1

3594,6

Consumo de alcohol

Si y aun consume

No

Ex consumidor

$\begin{array}{rr}28 & 9,0 \\ 5 & 5,2\end{array}$

9,0
5,2

28491,0

$92 \quad 94,8$

$1 \quad 11,1$

$8 \quad 88,9$

Actividad principal de $\quad 1$

su vida diaria*

1
2

18

\section{9,4}

174

90,6

$9 \quad 5,5$

155

94,5

$0,170^{* *}$

3

4

$7 \quad 14,0$

$43 \quad 86,0$

$0 \quad 0,0$

12100,0

Ver Televisión a diario

$$
\begin{aligned}
& \text { Si } \\
& \text { No }
\end{aligned}
$$

$12 \quad 12,2$

86

298

87,8

93,1

$0,547^{* *}$

$0,334^{* *}$

$22 \quad 6,9$

0,089*

1. Sentado/a la mayor parte de la jornada

2. Caminando, caminando llevando algún peso, efectuando desplazamientos frecuentes

3. De pie la mayor parte de la jornada sin efectuar grandes desplazamientos o esfuerzos

4. Realizando tareas que requieran gran esfuerzo físico

* Prueba Chi cuadrado;

** Prueba exacta de Fisher 
hallazgo es semejante al de varias investigaciones hechas en Colombia en diferentes universidades, que han encontrado cifras que van desde el $50,6 \%$ hasta incluso $77,8 \%$ que indican que los universitarios no realizan ninguna actividad física o desarrollan actividades de baja intensidad ${ }^{15,25,34}$. Este mismo comportamiento se ha evidenciado en otros países ${ }^{35}$. Igualmente, en Colombia, según la ENSIN 2010, tan solo el 19,9\% (mujeres 13,8\%; hombres 28,2\%) de personas de 18 a 64 años cumplen con las recomendaciones de actividad física durante el tiempo libre ${ }^{8}$.

Esta baja frecuencia de actividad física como parte de los comportamientos de salud de los universitarios podría estar determinada por causas multifactoriales ${ }^{35}$. Según lo hallado por Varela, la "pereza" puede ser una de las principales razones para no realizar actividad física entre los estudiantes, quienes se sienten a gusto con su estilo de vida sedentario ${ }^{25}$. Otros factores señalados como condicionantes de la inactividad física son la falta de tiempo y el apoyo social ${ }^{10,36,37}$. Cabe mencionar que el hábito de ver televisión en los universitarios se suma a la adquisición de estilos de vida poco activos y alteraciones en el peso corporal ${ }^{17,38}$.

\section{Antropometría}

En los resultados de IMC, nuestras cifras son menores a los datos de EP reportados a nivel nacional y local, según las cuales, para el país en el grupo de 18-32 años el EP fue de $34,3 \%^{8}$ y, para Medellín esta cifra alcanzó un $29,8 \%$ en el grupo de 18 a 30 años $^{9}$. Del mismo modo, otros estudios realizados en universitarios en Colombia ${ }^{15}$ y países como Chile ${ }^{36}$ y México ${ }^{39}$ han encontrado también mayores porcentajes de EP. Cabe mencionar que, en éste último, el EP se asoció con el tabaquismo, pues los alumnos que no fumaban tuvieron un IMC mejor comparado con aquellos que si fumaban ${ }^{39}$.

Sin embargo, nuestros hallazgos por EP son comparables al estudio realizado en universitarios de Murcia (España), cuyos valores medios del IMC estuvieron entre $23,9 \pm 3,8$ y $21,4 \pm 2,8 \mathrm{~kg} / \mathrm{m}^{2}$ en hombres y mujeres, respectivamente, con diferencias significativas por sexo $(\mathrm{p}<0,05)$, y una prevalencia global de $18,7 \%$ de $\mathrm{EP}^{40}$. Así mismo, es de resaltar que en todos los estudios mencionados, exceptuando el estudio nacional ${ }^{8}$, se evidenció, al igual que nuestros resultados, mayor frecuencia de EP en el sexo masculino.

Para el riesgo cardiovascular a partir del PC, la prevalencia encontrada en este estudio fue de $4,5 \%$ para los hombres y $9,1 \%$ para las mujeres, cifras bastante menores comparadas con las encontradas para el grupo de 18 a 32 años de la ENSIN 2010 (21,1\% hombres y 39,3\% mujeres $)^{8}$. No obstante, evidenciamos mayor prevalencia en el grupo de las mujeres, lo que es coincidente con la ENSIN 2010 y el estudio de Padilla y cols, $(24.4 \%$ mujeres versus $7,3 \%$ hombres) ${ }^{14}$.

El hecho de que la frecuencia de obesidad abdominal resulte mayor entre las mujeres, puede deberse a la realización de actividades de mayor esfuerzo físico entre los hombres; situación que se ha documentado en estratos socioeconómicos bajos y medios bajos, que son los que constituyen el mayor porcentaje de población de este estudio. Así mismo, las mujeres dedican mayor tiempo a realizar actividades sedentarias y menor práctica de ejercicio físico ${ }^{8}$.

Además, el estudio CARMELA sugiere que, en ciudades de Índice de Desarrollo Humano medio, como es el caso de las urbes colombianas, las mujeres de clase social baja tienen más obesidad abdominal, la cual se ha relacionado con la condición de ser madre y la depresión asociada al bajo nivel socioeconómico ${ }^{41}$.

En cuanto al riesgo de adiposidad, se observó en nuestros resultados que la totalidad de las personas que fueron clasificadas en obesidad por IMC se clasificaron en riesgo de enfermedad cardiovascular, así como el 32,2\% de los estudiantes con sobrepeso. Esto preocupa dada la condición de adulto joven de nuestra población objetivo, pues en diversos estudios se ha establecido la asociación entre alteraciones por EP en el estado nutricional por indicadores antropométricos con el posterior desarrollo de problemas de salud tal cómo las enfermedades crónicas no transmisibles ${ }^{4,42}$, situación que además se produce a una mayor velocidad de cambio en los países en vías de desarrollo y en el que interviene una combinación de múltiples factores además del IMC y el PC².

En esta muestra, a pesar que no se encontraron diferencias estadísticamente significativas, se observó que aquellos estudiantes con hábitos saludables respecto al no consumo de alcohol o tabaco alguna vez en su vida, presentaron las menores frecuencias de riesgo.

Finalmente, con respecto a la adiposidad corporal, nuestros resultados coinciden con lo reportado en un estudio chileno realizado en dos cohortes (2009 y 2014) de jóvenes universitarios, en la cual se evidenció para ambas mediciones, una mayor adiposidad corporal en los hombres, así mismo se observó un rápido aumento del indicador en este grupo en relación a las mujeres ${ }^{43}$.

Dentro de las limitaciones de este estudio, es importante indicar que el grupo poblacional en el cual está basado este artículo, no corresponde a una muestra representativa ni aleatoria, por la cual la generalización de los hallazgos debe circunscribirse a los participantes. Además, el tipo de estudio transversal no permite establecer causalidad.

\section{CONCLUSIONES}

Un porcentaje considerable de los universitarios participantes en este estudio, presentó exceso de peso establecidos a partir de su clasificación antropométrica, el cual se asoció con el sexo del estudiante y fue mayor en los hombres.

Aunque no se evidenciaron asociaciones del exceso de peso con las variables sociodemográficas y estilos de vida estudiadas, es importante que en los espacios universitarios se promuevan acciones que incentiven el empoderamiento de los estudiantes hacia estilos de vida saludable, esto incluye medidas que desincentiven factores que favorezcan el desarrollo temprano de enfermedades crónicas no 
transmisibles como consumo de bebidas alcohólicas, uso de tabaco/cigarrillo y sedentarismo.

\section{RESUMEN}

Introducción: Los estudiantes universitarios son particularmente vulnerables a una inadecuada nutrición, con la subsecuente afectación en su estado nutricional y de salud. Objetivo: Investigar la frecuencia de exceso de peso (EP) y algunos de sus factores asociados en estudiantes de la Universidad de Antioquia-Colombia. Métodos: Estudio transversal en 424 estudiantes, ambos sexos, con edades de 21,4 $\pm 4,0$ años. El EP se diagnosticó según índice de masa corporal $(I M C) \geq 25 \mathrm{~kg} / \mathrm{m}^{2}$; el riesgo de adiposidad central (RAC) como perímetro de cintura (PC) $\geq 80 \mathrm{~cm}$ en mujeres $y \geq 90 \mathrm{~cm}$ en hombres. Se midieron variables sociodemográficas y asociadas con el estilo de vida (EV). Se empleó estadística descriptiva, prueba $\mathrm{Chi}^{2}$ y $\alpha=0,05$. Resultados: El $17,1 \%$ de los estudiantes presentó EP, mayor en hombres que en mujeres (22,6\% vs $14,0 \%)$; un $8,1 \%$ mostró RAC, mayor en mujeres (9,1\% vs 6,5\%). El IMC se asoció con el sexo $(p=0,001)$. El RAC no mostró asociación con las características sociodemográficas ni de EV. Conclusión: Un porcentaje considerable de los universitarios presentó exceso de peso establecidos a partir de IMC, lo cual fue asociado al sexo del estudiante. No obstante, es importante promover espacios universitarios que propendan por estilos de vida saludables.

Palabras clave: obesidad, Sobrepeso, Circunferencia de la Cintura, índice de masa corporal, universitarios.

\section{BIBLIOGRAFÍA}

1. World Health Organization. Obesity and overweight [Internet]. WHO Media centre; 2016. [cited oct 2014]. http://www. who.int/mediacentre/factsheets/fs311/en/

2. Popkin B, Adair L, Wen S. Now and then: The Global nutrition transition: The pandemic of obesity in developing countries. Nutr Rev 2012; 70(1): 3-21.

3. World Health Organization. Diet, nutrition, and the prevention of chronic diseases: Report of a WHO Study Group (WHO Technical Report Series 797) [Internet]. Geneva: WHO; 2003 [cited oct 2014]. http://www.who.int/nutrition/publications/ obesity/WHO_TRS_797/en/

4. Prospective Studies Collaboration, Whitlock G, Lewington $S$, Sherliker P, Clarke R, Emberson J, et al. Body-mass index and cause-specific mortality in 900000 adults: collaborative analyses of 57 prospective studies. Lancet 2009; 373(9669): 1083-1096.

5. World Health Organization [Internet]. Social determinants of health: 2005-2008. WHO; 2016 [cited oct 2014] http://www. who.int/social_determinants/thecommission/finalreport/ key_concepts/en/

6. Dinsa GT, Goryakin Y, Fumagalli E, Suhrcke M. Obesity and socioeconomic status in developing countries: A systematic review. Obes Rev 2012; 13(11): 1067-1079.

7. González-Zapata LI, Estrada-Restrepo A, Álvarez-Castaño LS, Álvarez-Dardet C, Serra-Majem L. Excess weight and economic, political, and social factors: an international ecological analysis. Cad Saude Pública 2001; 27(9): 1746-1756.

8. Ministerio de la Protección Social, Instituto Nacional de Salud,
Instituto Colombiano de bienestar familiar. National Survey of Nutrition Status in Colombia (ENSIN) 2010. 1 Ed. Bogotá, D.C: Da Vinci Editores \& CIA. S N C; 2011.

9. Álvarez $L S$, Mancilla $L P$, González $L I$, Isaza UA. Food and nutritional profile of Medellín 2010. Medellín: Alcaldía de Medellín, University of Antioquia; 1 CD-ROM: sound, color. 4 3/4 en español. 2010

10. Downes L. Physical Activity and Dietary Habits of College Students. The Journal for Nurse Practitioners 2015; 11(2): 192-198.

11. Ledo-Várela $T$, Luis Román D, González-Sagrado M, Izaola Jauregui $O$, Conde Vicente $R$, Aller de la Fuente R. Nutritional characteristics and lifestyle in university students. Nutr Hosp 2011; 26(4): 814-818.

12. Ward H, Tarasuk V, Mendelson M, McKeown-Eyssen G. An exploration of socioeconomic variation in lifestyle factors and adiposity in the Ontario Food Survey through structural equation modeling. Int J Behav Nutr Phys Act 2007; 4: 8.

13. Lorenzini $R$, Betancur-Ancona D, Chel-Guerrero L, SeguraCampos M, Castellanos-Ruelas A. Nutritional status of university students from México in relation with their lifestyle. Nutr Hosp 2015; 32(1): 94-100.

14. Padilla-García Cl, Jaimes-Valencia M, Fajardo-Nates S, RamosFranco A. Cardiovascular risk factors of college students. MedUNAB 2014; 17(2): 81-90.

15. Rangel Caballero L, Rojas Sánchez Z, Gamboa Delgado E. Overweight and Obesity in Colombian college students and its association with physical activity. Nutr Hosp 2015; 31(2): 629-636.

16. Pengpida S, Peltzer K. Prevalence of overweight/obesity and central obesity and its associated factors among a sample of university students in India. Obesity Research \& Clinical Practice 2014; 8: e558-e570

17. World Health Organization. The world health report 2002 - Reducing Risks, Promoting Healthy Life [Internet] France: WHO; 2002. 165 p. [cited oct 2014]. http://www.who.int/ whr/2002/en/whrO2_en.pdf?ua=1

18. Colombia's Drug Observatory. Colombia Drug Report. 1 Ed [Internet] Bogotá: ODC; 2015. https://www.odc.gov. co/Portals/1/publicaciones/pdf/odc-libro-blanco/OD01003 11215_reporte_de_drogas_de_colombia.pdf

19. Ministry of Justice and Law, Colombia's Drug Observatory, Ministry of Health and Social Welfare. National study of the use of psychoactive substances in Colombia - 2013. [Internet] Bogotá: ALVI Impresores S.A.S; 2014. [cited nov 2014]. https://www.unodc.org/documents/colombia/2014/ Julio/Estudio_de_Consumo_UNODC.pdf

20. District Health Secretary. Study of Psychoactive substance use in Medellín, Metropolitan area and rest of Antioquia - 2009. [Internet] Medellín; 2009. http://www.odc.gov. co/Portals/1/publicaciones/pdf/consumo/estudios/locales/ CO031052009-estudio-consumo-sustancias-psicoactivasmedellin-area-metropolitana-resto-antioquia-2009.pdf

21. Montoya Vásquez E, Cunningham J, Brands B, Strike C, MiottoWrigh M. Consumption and perceived use of licit and illicit drugs among university students, Antioquia, Medellin, Colombia. Rev Lat Am Enfermagem 2009; 17: 886-892.

22. Muñoz L, Barbosa C, Bríñez A, Caycedo C, Méndez M, Oyuela $R$. Elements for prevention programs in alcohol consumption in university students. Universitas Psychologica 2012; 11(1): 131-145.

23. Illegal Anti-Drug Program of the Andean Community PRADICAN II. Andean Epidemiological Study on Drug Use 
in the University Population Report Colombia 2012. [Internet] Lima: OHQUIS DESIGN E.I.R.L; enero 2013. 114 p. [cited nov 2014] http://www.odc.gov.co/Portals/1/publicaciones/ pdf/destacados/CO03542012-ii-estudio-epidemiologicoandino-sobre-consumo-drogas-poblacion-universitariainforme-colombia-2012-.pdf

24. Lema Soto LF, Salazar Torres I, Varela Arévalo M, Tamayo Cardona J, Rubio Sarria A, Botero Polanco A. Behavior and health of college students: satisfaction with lifestyle. Pensamiento Psicológico 2009; 5(12): 71-88.

25. Varela MT, Duarte C, Salazar IC, Lema LF, Tamayo L. Physical activity and sedentary behavior in college youth from Colombian cities: practices, motivations, and resources. Colomb Med 2011; 42(3): 269-277.

26. Ministry of social protection. Resolution 00002121 of 2010, which adopts the Growth Patterns published by the World Health Organization-WHO in 2006 and 2007 for children and adolescents from 0 to 18 years of age and other dispositions are dictated. [Internet] Colombia: Ministry of social protection; 2010. [cited nov 2014]. https://www.minsalud.gov.co/ Normatividad_Nuevo/Resoluci\%C3\%B3n\%202121\%20 de\%202010.pdf

27. World Health Organization. BMI classification. [Internet] WHO. http://apps.who.int/bmi/index.jsp? introPage=intro_3. html

28. Alberti K, Robert E, Grundy S, ZimmetP, CleemanJ, DonatoK. et al. Harmonizing the Metabolic Syndrome: A Joint Interim Statement of the International Diabetes Federation Task Force on Epidemiology and Prevention; National Heart, Lung, and Blood Institute; American Heart Association; World Heart Federation; International Atherosclerosis Society; and International Association for the Study of Obesity. Circulation 2009; 120: 1640-1645.

29. Rodríguez Gázquez $M$, Pineda Botero $S$, Vélez Yépes $L$. Tobacco consumption characteristics in nursing students of the University of Antioquia (Colombia). Investigación y Educación en Enfermería 2010; 28(3): 370-383.

30. World Health Organization. WHO report on the global tobacco epidemic 2009 [Internet]. Geneve: World Health Organization; 2009. http://apps.who.int/iris/ bitstream/10665/44420/1/9789243563916_spa.pdf

31. Salcedo Monsalve A, Palacios Espinosa X, Fernanda Espinosa $F$. Alcohol consumption in young college students. Avances en Psicología Latinoamericana/Bogotá (Colombia) 2011; 29(1): 77-97.
32. National Institutes of Health, NIH. Alcohol A Women's Health Issue. United States; 2005. https://pubs.niaaa.nih. gov/publications/WomenSpanish/Women_Spanish.pdf

33. Traversy G, Chaput JP. Alcohol Consumption and Obesity: An Update. Curr Obes Rep 2015; 4: 122-130.

34. Díaz Cárdenas S, González Martínez F, Arrieta Vergara K. Physical activity levels associated with sociodemographic, anthropometric and behavioral factors in university students of Cartagena (Colombia). Salud Uninorte 2014; 30(3): 405417.

35. Musaiger AO, Al-Khalifa F, Al-Mannai M. Obesity, unhealthy dietary habits and sedentary behaviors among university students in Sudan: growing risks for chronic diseases in a poor country. Environ Health Prev Med 2016; 21(4): 224-230.

36. Rodríguez F, Palma X, Romo A, Escobar D, Aragú B, Espinoza $L$, et al. Eating habits, physical activity and socioeconomic level in university students of Chile. Nutr Hosp 2013; 28(2): 447-455.

37. Martinez YTS, Harmon BE, Nigg CR, Bantum EO, Strayhorn S. iet and Physical Activity Intervention Strategies for College Students. Health Behav Policy Rev 2016; 3(4): 336-347.

38. Martínez-Moyá M, Navarrete-Muñoz E, García de la Hera M, Giménez-Monzo D, González-Palacios S, Valera-Gran D. Association between hours of television watched, physical activity, sleep and excess weight among young. Gac Sanit 2014; 28(3): 203-208.

39. Lorenzini $R$, Betancur-Ancona D, Chel-Guerrero L, SeguraCampos $M$, Castellanos-Ruelas A. Nutritional status of university students from México in relation with their lifestyle. Nutr Hosp 2015; 32(1): 94-100.

40. Cutillas AB, Herrero E, de San Eustaquio A, Zamora S, Pérez-Llamas F. Prevalence of underweight, overweight and obesity, energy intake and dietary caloric profile in university students from the region of Murcia (Spain). Nutr Hosp 2013; 28(3): 683-689.

41. Pramparo P, Boissonnet C, Schargrodsky H. Evaluation of Cardiovascular Risk in Seven Cities in Latin America: The Main Conclusions of the Carmela Study Rev Argent Cardiol 2011; 79(4): 377-382.

42. Haslam DW, James WP. Obesity. Lancet 2005; 366: 1197209.

43. Contreras Mellado V, Vilchez Avaca C, Gómez-Campos R, Luarte Rocha C, Cossio-Bolaños M. Trends to increased body fat and blood pressure of university students in two cohorts (2009-2014). Nutr Hosp 2015; 32(6): 2551-2558. 